\title{
Utilização de Stent para Tratamento de Dissecção Coronária Iatrogênica
}

\author{
Ronaldo Tadeu Copini, Claudia Maria Rodrigues Alves, Francisco de Assis Costa, \\ José Augusto Marcondes de Souza, João Lourenço Villari Herrmann
}

São Paulo, SP

\begin{abstract}
A dissecção coronária iatrogênica é rara, entretanto, apresenta alta mortalidade e morbidade.

Diante de uma oclusão coronária aguda é fundamental a restauração imediata da perviabilidade do vaso e, com isso, limitar a extensão e a duração da isquemia. Neste contexto o procedimento usual tem sido a revascularização miocárdica de emergência, precedido pela colocação de um balão intra-aórtico. A principal desvantagem desta abordagem é o tempo necessário para reunir uma equipe cirúrgica.

Os autores apresentam caso de uma paciente com dissecção iatrogênica da artéria circunflexa durante o cateterismo diagnóstico, que foi solucionada através da implantação de um stent coronário, com ótimo resultado. Esta técnica apresenta vantagens em termos de velocidade de reperfusão e disponibilidade em centros que realizarão o cateterismo diagnóstico sem cobertura cirúrgica local.
\end{abstract}

\section{Stenting for Iatrogenic Coronary Dissection}

Catheter-induced coronary dissection is rare but has a significant mortality and morbidity.

The ideal management of acute coronary occlusion, whatever the cause, is the prompt restoration of the vessel patency to limit the extent and duration of ischemia. In the setting of dissection during angiography the usual approach to myocardial salvage has been emergency aortocoronary bypass surgery preceded by insertion of an intra-aortic balloon. The main disadvantages of this approach is the delay in performing emergency surgery, mainly in centers without surgical back-up on site.

The authors present a case of an elderly woman with left circumflex artery dissection caused by the catheter during coronary angiography. This problem was successfully managed using an intracoronary stent. This technique has potential advantages in terms of the speed of reperfusion and availability in centers performing diagnostic angiography without surgical back-up on site.

Arq Bras Cardiol, volume 70 (n' 1), 55-57, 1998

A dissecção coronária provocada pelo cateterismo cardíaco diagnóstico é uma complicação rara e grave com uma incidência de $0,01 \%$ a $0,41 \%{ }^{1}$, sendo geralmente resultado de trauma direto à intima arterial. Dependendo de sua gravidade, rapidamente, se estabelece oclusão proximal. $\mathrm{O}$ tratamento imediato para restabelecimento da perviabilidade vascular é mandatório, limitando a duração e extensão da isquemia.

Na sala de hemodinâmica, a utilização de cateteres de perfusão e balão intra-aórtico, quando há instabilidade hemodinâmica, ajudam na estabilização do paciente para

Escola Paulista de Medicina - UNIFESP

Correspondência: Ronaldo Tadeu Copini - Escola Paulista de Medicina - UNIFESP

- Rua Botucatu, 740 - 04023-062 - São Paulo, SP

Recebido para publicação em 16/9/97

Aceito em 17/10/97 encaminhamento cirúrgico. Relatamos um caso de dissecção severa tratada com angioplastia e stent com excelente resultado.

\section{Relato do Caso}

Paciente do sexo feminino, 78 anos, encaminhada para cinecoronariografia eletiva, ambulatorial, com antecedente de hipertensão arterial leve e precordialgia aos esforços, há cerca de um ano, sendo que, nos últimos dois meses, tornou-se mais severa em frequiência e duração. OECG evidenciava apenas sinais de hipertrofia ventricular leve.

O cateterismo cardíaco foi realizado através de acesso femoral direito, pela técnica de Seldinger. Com o cateter Judkins 3,5-6F foi cateterizada a artéria coronária esquerda sob monitorização pressórica. Duas injeções em projeções ortogonais revelaram artéria livre de processos obstrutivos, com um tronco muito curto, e injeções semi-seletivas na ar- 


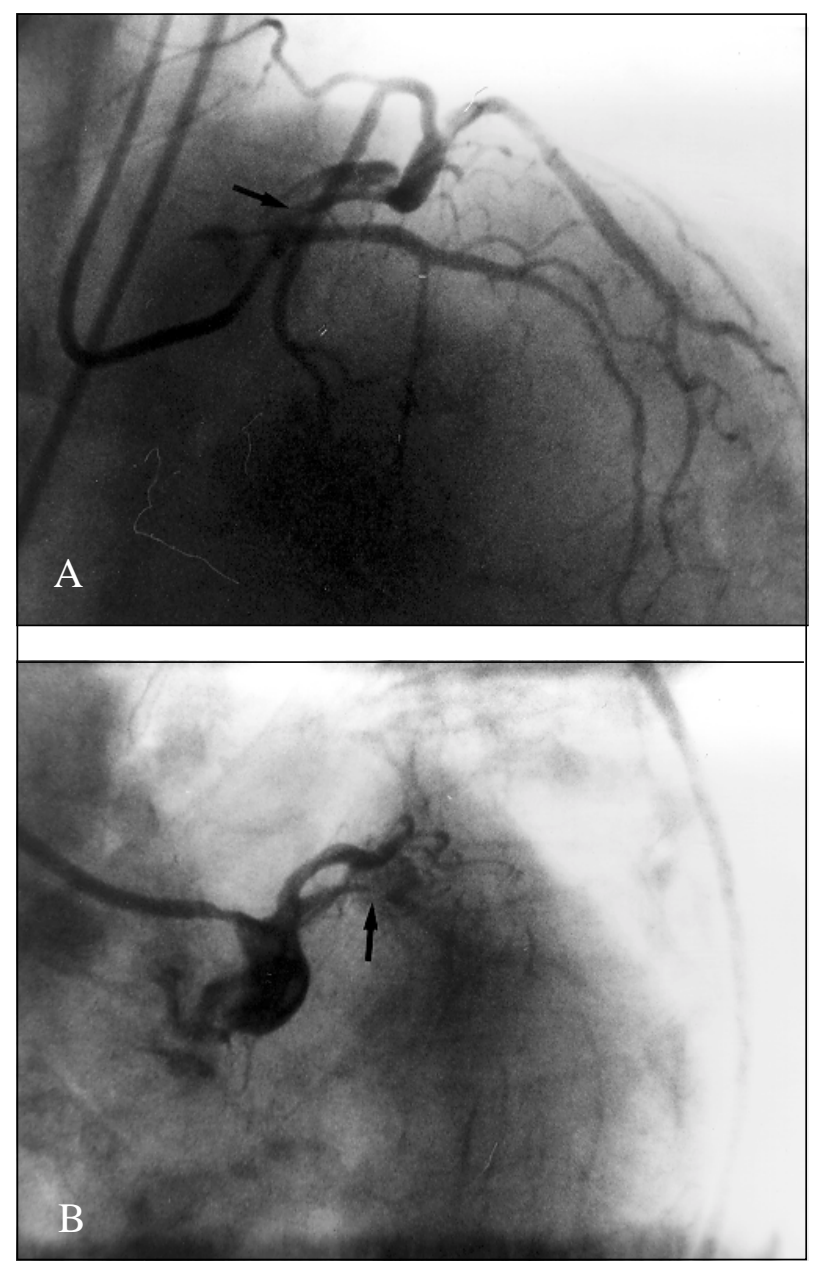

Fig. 1 - A) Coronariografia esquerda em projeção PA cranial. A seta indica extensa dissecção na porção proximal da artéria circunflexa. A artéria descendente anterior está preservada; B) após poucos minutos, a coronariografia em OAE já demonstra oclusão total proximal da artéria circunflexa (seta).

téria circunflexa $(\mathrm{Cx})$, que era de grande calibre, fornecendo grande ramo marginal e segundo ramo póstero-lateral. Durante a terceira injeção, observou-se extensa infiltração de contraste na parede da $\mathrm{Cx}$, comprometendo sua porção proximal (fig. 1), com prejuízo severo do fluxo e que evoluiu, rapidamente, para oclusão arterial aguda. A origem e fluxo da artéria descendente anterior (ADA) estavam preservados. A paciente passou a apresentar precordialgia severa, palidez, sudorese e a monitorização eletrocardiográfica mostrou sinais de infarto em instalação.

A angioplastia arterial coronária com cateter de perfusão foi iniciada, imediatamente. Conseguiu-se fácil progressão do fio-guia 0,014" J Ex Hannibal $315 \mathrm{~cm}$ (Schneider Inc) posicionado no grande ramo marginal da Cx. Cateter SpeedFlow 3,5x30mm (Schneider Inc) foi posicionado após cerca de $5 \mathrm{~min}$ de oclusão no ponto de retenção de contraste, obtendo-se imediata reperfusão e fluxo normal na ADA cuja emergência era contígua à lesão. Após 15 min de insuflação, nova coronariografia demonstrou perviabilidade arterial, logo seguida por nova oclusão total da artéria. Considerando a grande importância da artéria e sua proximidade com o tronco da ACE, optou-se por imediata liberação de stent. Um microstent II 3,5x39mm (Arterial VascularEngineering, Inc) foi liberado, ainda com o fio-guia posicionado no ramo marginal. Considerando-se a gravidade do caso, decidimos enfrentar a possibilidade de oclusão do ramo atrioventricular da $\mathrm{Cx}$, que emergia da porção a ser coberta pelo stent. A pós-dilatação com cateter mongoose 3,5x20mm (Schneider Inc) foi realizada em toda a extensão do stent até 17 atm de pressão. Após a liberação do stent, houve completa remissão dos sintomas e normalização eletrocardiográfica.

A quantificação coronária automática (CMS - MEDIS) realizada na coronariografia de controle, após retirada do fio-guia, revelou diâmetro de referência de 3,62mm e diâmetro da obstrução de 3,38mm ( $1 \%$ de estenose do diâmetro). As emergências da ADA e porção atrioventricular da $\mathrm{Cx}$ estavam livres de obstrução e com fluxo normal (fig. 2).

A paciente foi mantida sob monitorização em UTI por 48h, anticoagulada por $48 \mathrm{~h}$ e recebeu alta após três dias, em uso de ácido acetil-salicílico e ticlopidina, não tendo ocorrido alteração eletrocardiográfica ou enzimática na evolução.

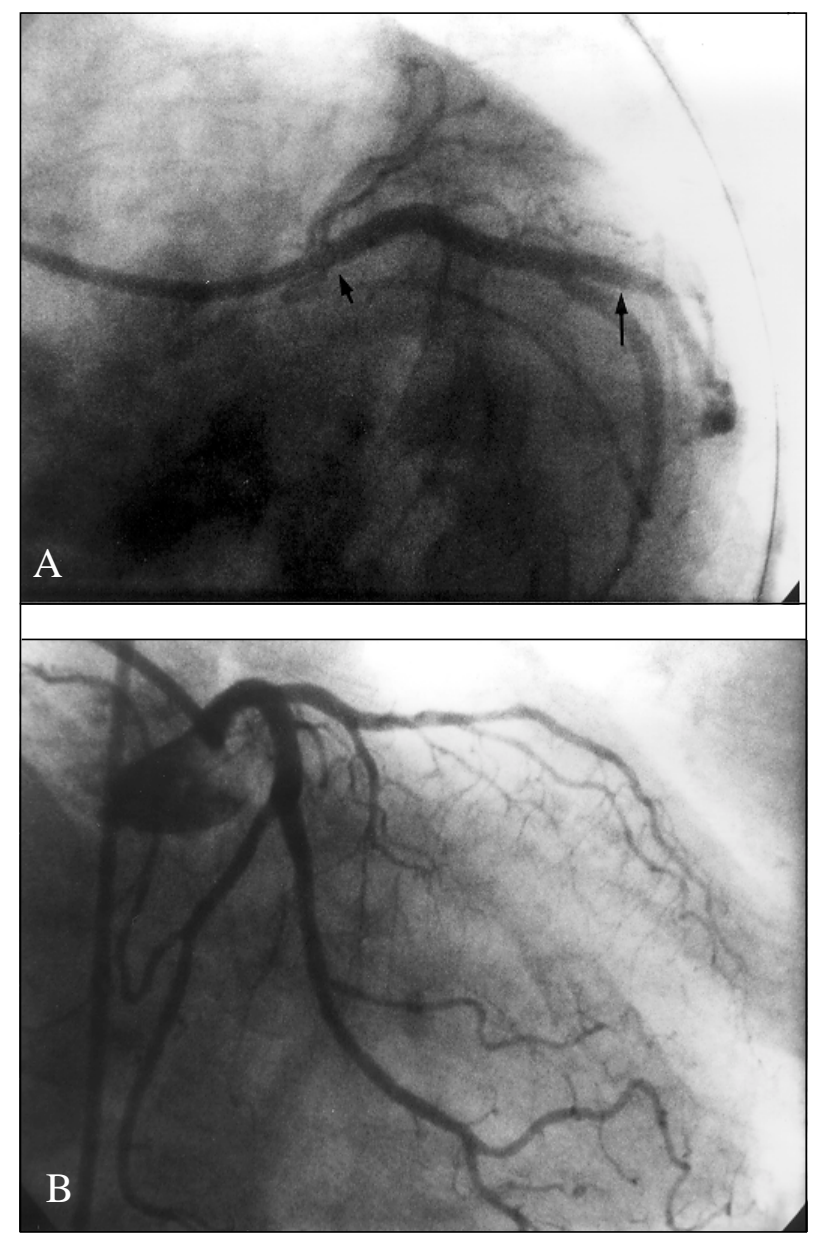

Fig. 2 - A) Coronariografia seletiva em artéria circunflexa em projeção OAE caudal, mostrando lúmen amplo e regular, com preservação da porção atrioventricular da artéria. As setas indicam a extensão do stent; B) coronariografia esquerda em projeção OAD. Resultado final da angioplastia. 


\section{Discussão}

A dissecção coronária provocada por cateter e relacionada a procedimentos diagnósticos, ou seja, excetuandose aquela relacionada à angioplastia, ocorre igualmente em ambos os sexos, não tem relação com a técnica - braquial ou femoral - e envolve predominantemente a artéria coronária direita $(67 \%)^{2}$. Quando a via femoral é a utilizada, há algumas evidências de que a dissecção é mais comum com cateteres mais finos $(0,67 \%$ para cateteres $6 \mathrm{~F}$ vs $0,04 \%$ para cateteres $8 \mathrm{~F})^{3}$. Algumas características dos cateteres $6 \mathrm{~F}$ como facilidade de penetração profunda na coronária e menor torque e estabilidade no óstio facilitam a injúria intimal ${ }^{4}$, especialmente, no ambiente de treinamento que caracteriza a instituição de ensino. Obviamente, a dissecção é ainda facilitada pela presença de aterosclerose.

Nas dissecções coronárias graves, o procedimento usual, principalmente no período anterior à utilização dos stents, é a revascularização de emergência devido a complexidade da sua apresentação e pela grande área de miocárdio em risco. Apesar dos bons resultados cirúrgicos ${ }^{5}$, o tempo necessário para se reunir uma equipe cirúrgica durante uma emergência e a morbidade do procedimento são pontos desfavoráveis na sua comparação com a angioplastia. Serviços de hemodinâmica não ligados a centros cirúrgicos representam outro ambiente onde a ATC deve ser o procedimento terapêutico utilizado.

O stent coronário já tem função estabelecida no tratamento da doença arterial coronária, tanto como escolha primária quanto no manuseio das complicações de angioplastia. No nosso caso, uma dissecção de grande proporção e extensão mal definida, comprometendo a origem da $\mathrm{Cx}$ e intimamente relacionada à emergência da $\mathrm{ADA}$, foi tratada com extrema facilidade e rapidez pela utilização de stent longo. Não foi observada qualquer dissecção residual.

A utilização de stents para os casos mais dramáticos de dissecção iatrogênica, quer como tratamento definitivo quer como ponte para posterior revascularização, deve se tornar uma rotina, uma vez que o procedimento mostra-se bastante simples e proporciona vantagens sobre o tratamento cirúrgico, como rapidez da revascularização, menor morbidade e economia de recursos ${ }^{6}$.

\section{Referências}

1. De Bono D - Complications of diagnostic cardiac catheterization . Results from 3401 patients in United Kingdom confidential enquires into cardiac catheter complications. Br Heart J 1993; 70: 297-300.

2. Mouse AP, Hardin NJ , Bowell N et al - Coronary artery disseccion secondary to coronary arteriography. Cathet Cardiovasc Diag 1981; 7: 283-96.

3. Prewitt KC,Zen B, Wortham DC et al - Increased risk of coronary artery disseccion during angiography whit 6F catheters. Angiology 1993; 44: 107-13.
4. Ellis SG, Pinto IM, McGullin MJ et al - Accuracy and reproducibility of quantitative coronary arteriography using 6 and 8 French catethers with cine angiographic aquisition. Cathet Cardiovasc Diag 1991; 22: 52-5.

5. Fronc HC, Mac Millan R, Kimberis D - Clinical case report - coronary artery disseccion. Angiology 1990; 41(10): 884-7.

6. Knight C, Stables R, Sigwart U - Emergency coronary artery stenting for coronary disseccion complicating diagnostic cardiac. Br Heart J 1995; 74: 199-201. 